\title{
Adesão à terapia em motricidade orofacial no ambulatório de Fonoaudiologia do Hospital das Clínicas da Universidade Federal de Minas Gerais
}

\author{
Adhesion to orofacial myology therapy at the ambulatory of \\ Speech and Language Pathology of the Hospital das Clinicas \\ of Universidade Federal de Minas Gerais
}

Suzana Raquel Lopes Marques ${ }^{1}$, Amélia Augusta de Lima Friche², Andréa Rodrigues Motta ${ }^{3}$

\begin{abstract}
RESUMO
Objetivo: Caracterizar o atendimento em motricidade orofacial no Ambulatório de Fonoaudiologia do Hospital das Clínicas da Universidade Federal de Minas Gerais quanto à adesão e sucesso da terapia, bem como comparar dados dos pacientes que buscaram atendimento ativamente no Ambulatório de Fonoaudiologia e dos pacientes encaminhados pelo Ambulatório do Respirador Oral da mesma instituição. Métodos: Foram investigados 90 prontuários de todos os pacientes em alta ou desligados, que buscaram tratamento pelos Ambulatórios de Fonoaudiologia (Grupo MO) e do Respirador Oral (Grupo RO). Os resultados foram analisados empregando-se os testes Mann-Whitney e Qui-quadrado (5\%). Resultados: Pelo cálculo da mediana, a duração da terapia foi equivalente a 6,0 meses, o número total de sessões foi igual a 17, o de atendimentos realizados 12 e o de faltas 4,5. Apenas 41,2\% dos pacientes realizava os procedimentos indicados pelo terapeuta. A conclusão do processo terapêutico foi majoritariamente o desligamento $(73,3 \%)$, sendo a maior causa o excesso de faltas $(24,4 \%)$. Os grupos diferiram quanto aos motivos de desligamento, havendo predomínio de faltas no grupo $\mathrm{RO}(\mathrm{p}<0,01)$ e espera por outros profissionais no Grupo $\mathrm{MO}(\mathrm{p}<0,01)$. Ocorreram associações significantes entre faltas e desligamentos $(\mathrm{p}<0,01)$ e adesão e desligamentos $(\mathrm{p}<0,01)$ no Grupo RO. Conclusões: As características pesquisadas foram predominantemente semelhantes nos grupos. Apesar de muitos pacientes seguirem as orientações total ou parcialmente, a adesão ficou prejudicada pelo excesso de faltas. Assim, o sucesso da terapia não foi alcançado na maioria dos casos.
\end{abstract}

Descritores: Fonoterapia; Terapia miofuncional; Anormalidades do sistema estomatognático; Respiração bucal; Resultado de tratamento; Pesquisa sobre serviços de saúde; Alta do paciente

\section{INTRODUÇÃO}

No contexto que se refere aos tratamentos de saúde, o termo adesão tem apresentado definições distintas. A adesão pode ser considerada como o quanto os pacientes são obedientes e

Trabalho realizado na Universidade Federal de Minas Gerais - UFMG - Belo Horizonte (MG), Brasil.

(1) Fonoaudióloga clínica - Belo Horizonte (MG), Brasil.

(2) Pós-graduanda (Doutorado) em Saúde Pública pela Universidade Federal de Minas Gerais - UFMG - Belo Horizonte (MG), Brasil; Professora assistente do Departamento de Fonoaudiologia da Universidade Federal de Minas Gerais - UFMG, Belo Horizonte (MG), Brasil.

(3) Pós-graduanda (Doutorado) em Distúrbios da Comunicação Humana pela Universidade Federal de São Paulo - UNIFESP - São Paulo (SP), Brasil; Professora assistente do Departamento de Fonoaudiologia da Universidade Federal de Minas Gerais- UFMG - Belo Horizonte (MG), Brasil.

Endereço para correspondência: Suzana Raquel Lopes Marques. R. Dr. Luiz Ratton, 108/202, Bl 1, Bairro Manacás, Belo Horizonte (MG), Brasil, CEP: 30840-600. E-mail: suzilps@yahoo.com.br

Recebido em: 22/9/2008; Aceito em: 19/8/2009 seguem as instruções, proibições e prescrições do profissional de cuidado à saúde ${ }^{(1-2)}$. Contudo, há definições mais amplas e conscientes da dinâmica do processo e que relacionam-se ao conceito de envolvimento ativo, voluntário e colaborativo do paciente em uma linha de comportamento mutuamente aceitável, com o objetivo de produzir os resultados terapêuticos ou preventivos desejados ${ }^{(2)}$. A adesão pode ser parcial, resultando do entendimento de que pode haver concordância e realização parcial do plano terapêutico proposto pelo profissional de saúde, aceitando que a adesão não deve ser analisada de forma dicotômica, considerando o paciente simplesmente como aderente ou não-aderente ${ }^{(1)}$.

Associada ao correto diagnóstico e seleção do tratamento, a adesão à terapia é necessária para melhorar a saúde ${ }^{(2)}$, uma vez que há relação direta entre comportamento de adesão e aprimoramento dos resultados em saúde ${ }^{(2-3)}$. Diante deste fato, estudos sobre a adesão, intervenções para aprimorar este aspecto e formas de auxiliar o paciente devem ser realizados ${ }^{(4)}$. 
A natureza dos determinantes da não adesão é complexa e ainda pouco compreendida ${ }^{(4-5)}$. Entretanto, algumas razões para a adesão ser inferior à ideal podem ser mencionadas: pacientes com vidas muito ocupadas; associação de várias, por vezes conflituosas, prioridades e dificuldades em se adaptar às novas rotinas propostas pelo tratamento ${ }^{(2)}$. Outro fator considerado como predisponente à não adesão ao tratamento é a depressão(6), sendo que a estrutura social e familiar do indivíduo também influenciam ${ }^{(7)}$. Além disso, os pacientes constroem seus próprios conceitos de adesão de acordo com questões pessoais e contexto social. Todos esses aspectos podem resultar em divergência de expectativas em relação à adesão entre o profissional de saúde e o paciente ${ }^{(2)}$.

Diante desta situação, o profissional de saúde deve investir em estratégias de promoção à adesão, consciente da existência da variabilidade deste aspecto, considerando a realidade de cada paciente e as possíveis mudanças de comportamento do sujeito ao longo do período de intervenção ${ }^{(2)}$. Considera-se relevante para que a eficácia da terapia seja alcançada o respeito à integralidade, princípio normativo do SUS, assim como a adequada relação entre profissionais de saúde e pacientes ${ }^{(8)}$. A atenção humanizada, em que a relação entre profissionais de saúde e usuários, assim como a comunicação (verbal ou não) efetiva entre as partes sejam valorizadas é considerada como um fator de extrema importância para a adesão do paciente ao tratamento $^{(9)}$. Medidas simples, como questionar diretamente o paciente quanto à adesão, observar a frequência às sessões e resposta ao tratamento, são eficazes para se detectar a maioria dos problemas ${ }^{(10)}$.

Até o presente momento, poucos estudos com o objetivo de quantificar os resultados da terapia têm sido conduzidos. Estas medidas são importantes para a prática clínica, apesar de muitos fonoaudiólogos acreditarem que sua aplicação seja difícil $^{(11)}$. Conhecer o tempo médio de fonoterapia para se conseguir resultados positivos em cada alteração trabalhada, contribui para que o fonoaudiólogo tente, a partir destes dados, aprimorar suas técnicas e reduzir o tempo de terapia. Ademais, se o paciente e sua família souberem que o tratamento será realizado em determinado período, investem e participam mais ativamente do processo terapêutico ${ }^{(12-13)}$. Vários estudos indicam ainda que a atuação interdisciplinar nas alterações do sistema estomatognático é extremamente importante para promover melhores resultados na reabilitação. A troca de informações entre os profissionais pode definir as possibilidades, implicações, limitações e a real necessidade de cada terapêutica, assim como favorecer a estabilidade dos resultados obtidos ${ }^{(14-18)}$.

Até o presente momento, não foi caracterizado o atendimento em motricidade orofacial referente aos distúrbios da respiração, mastigação, deglutição e fala oferecido no ambulatório de Fonoaudiologia do HC/UFMG. A partir da coleta e interpretação de fatos que se repetem torna-se mais fácil estabelecer condutas terapêuticas com maior eficácia e comprovar sua validade ${ }^{(19)}$, o que ressalta a importância de registrar, arquivar e computar as informações progressivamente coletadas nos serviços. A análise dos registros permite aos fonoaudiólogos adotarem ações mais eficazes e coerentes com as necessidades locais, bem como o aprimoramento da dinâmica de atendimento ${ }^{(20)}$.
Assim, o levantamento de dados quantitativos acerca do tratamento oferecido e seus resultados pode ser uma ferramenta útil para que se consiga repensar a prática em motricidade orofacial. Desta forma, será possível elaborar estratégias para a ampliação da adesão à terapia fonoaudiológica, considerada aqui como a frequência às sessões e realização dos procedimentos indicados pelo terapeuta responsável, assim como promover maior percentual de sucesso da terapia fonoaudiológica, ou seja, maiores índices de adequação/ adaptação das estruturas e funções miofuncionais orofaciais dos pacientes vinculados ao serviço mencionado.

Portanto, os objetivos deste estudo foram caracterizar o atendimento em motricidade orofacial no ambulatório de Fonoaudiologia do Hospital das Clínicas da Universidade Federal de Minas Gerais (HC/UFMG) quanto à adesão e sucesso da terapia, bem como comparar dados dos pacientes que buscaram atendimento fonoaudiológico ativamente no Ambulatório de Fonoaudiologia do HC/UFMG e dos pacientes encaminhados pelo Ambulatório do Respirador Oral da mesma instituição.

\section{MÉTODOS}

Previamente à realização da investigação, o projeto da pesquisa foi aprovado pelo Comitê de Ética em Pesquisa da Universidade Federal de Minas Gerais (UFMG), registrado sob o número ETIC 73/07, conforme a resolução 196/96 da Comissão Nacional de Ética e Pesquisa (CONEP).

Este estudo transversal foi realizado por meio de pesquisa com dados secundários. Foram coletadas informações referentes ao atendimento em motricidade orofacial, especificamente dos casos com diagnóstico de distúrbios da respiração, mastigação, deglutição e fala de origem músculoesquelética, dos prontuários do Ambulatório de Fonoaudiologia do Hospital das Clínicas da UFMG.

$\mathrm{O}$ ambulatório de Fonoaudiologia oferece atendimento nas áreas de linguagem, reabilitação auditiva, voz e motricidade orofacial, enquanto o ambulatório do Respirador Oral da mesma instituição constitui um grupo de referência em avaliação multidisciplinar do respirador oral e conta com a atuação de profissionais das áreas de Otorrinolaringologia, Fonoaudiologia, Ortodontia e Alergologia. Quando é confirmado o diagnóstico de respiração oral e verificada por meio da avaliação fonoaudiológica a presença de comprometimento do sistema estomatognático, o paciente é encaminhado para atendimento em grupo no Ambulatório de Fonoaudiologia.

Inicialmente considerou-se os prontuários de todos os pacientes em alta fonoaudiológica ou desligados, que buscaram tratamento tanto pelo próprio ambulatório de Fonoaudiologia (Grupo MO) quanto pelo ambulatório do Respirador Oral (Grupo RO), totalizando 157 prontuários de pacientes entre três e 46 anos, todos cadastrados nas Unidades Básicas de Saúde de Belo Horizonte, Minas Gerais.

Posteriormente, foram excluídos do estudo 45 prontuários, referentes aos pacientes com distúrbio miofuncional orofacial e cervical associado a desvios fonológicos. Além disso, para garantir a comparabilidade entre o Grupo MO e o Grupo RO 
foram excluídos 21 prontuários do Grupo MO. Estes registros são relativos aos pacientes com idade entre 13 e 46 anos, tendo sido eliminados do estudo uma vez que o Ambulatório do Respirador Oral atende apenas a faixa etária de três a 12 anos de idade.

Mais um prontuário precisou ser excluído da amostra, uma vez que se caracterizou como um outlier, em decorrência do tempo de terapia ter sido muito superior em relação aos demais pacientes do Ambulatório. Portanto, no presente estudo, foram investigados 90 prontuários, sendo 56 pacientes do gênero masculino e 34 do feminino, compreendendo o período desde a inauguração do serviço, em julho de 2003, a julho de 2007.

Os prontuários foram consultados dentro do ambulatório de Fonoaudiologia, no período de março a julho de 2007. Os dados coletados foram transcritos para um roteiro, elaborado pelas autoras (Anexo 1), que constou de dois itens: dados sobre a adesão ao tratamento e sucesso da terapia fonoaudiológica.

As informações acerca da adesão ao tratamento, quanto à data de início e término da terapia, número total de sessões, de atendimentos, de faltas e de atrasos, foram obtidas nas folhas de registro, as quais detalham cada sessão de terapia. Os dados relativos à realização dos procedimentos indicados pelo terapeuta responsável foram coletados no primeiro e no último relatórios de evolução. Ressalta-se que este item foi desconsiderado quando o número de sessões a que o paciente compareceu era inferior a cinco, por se considerar que este número não é expressivo o suficiente para analisar a adesão do indivíduo à terapia. Em relação ao sucesso da terapia, considerou-se que este foi alcançado quando o paciente obteve alta fonoaudiológica com adequação/adaptação das estruturas e funções miofuncionais orofaciais ou alta temporária. Para a obtenção dos dados acima, foram consultados os relatórios de alta ou desligamento.

As informações obtidas foram analisadas estatisticamente. Caracterizou-se o serviço quanto aos aspectos estudados tendo-se comparado os resultados encontrados em relação ao atendimento em motricidade orofacial dos pacientes que buscaram atendimento ativamente no ambulatório de Fonoaudiologia (Grupo MO) e dos pacientes indicados para fonoterapia pelo Ambulatório do Respirador Oral (Grupo RO), ambos pertencentes ao HC/UFMG. Para tanto, empregaram-se os testes não-paramétricos de Mann-Whitney, para as variáveis quantitativas, e Qui-quadrado, para as variáveis categóricas. Para análise das variáveis categóricas foram utilizadas também medidas de tendência central e dispersão. Considerou-se na interpretação dos resultados o nível de significância de 5\%.

$\mathrm{Na}$ análise dos dados referentes à adesão à terapia, as variáveis contínuas foram posteriormente transformadas em categóricas para apreciação mais ampla dos achados. Quanto à duração da terapia, ao número total de sessões, de atendimentos e de faltas, estabeleceu-se como ponto de corte a mediana obtida para cada uma das variáveis.

\section{RESULTADOS}

Verificou-se no Grupo MO a presença de 21 indivíduos do sexo masculino $(75 \%)$ e sete do feminino $(25 \%)$ e, no Grupo RO, 35 indivíduos do sexo masculino (56,4\%) e 27 do feminino (43,6\%). Não foi aferida diferença significante, pelo teste Qui-quadrado entre os grupos quanto ao gênero $(\mathrm{p}=0,09)$.

Quanto à idade, no Grupo MO observou-se a ocorrência de seis indivíduos com faixa etária de três a cinco anos $(21,4 \%), 12$ entre seis e oito anos $(42,9 \%)$ e dez entre nove e 12 anos $(35,7 \%)$. A média de idade foi de 7,5 $\pm 2,7$ anos, sendo a mediana equivalente a 7 anos. No Grupo RO, houve oito indivíduos com faixa etária de três a cinco anos (12,9\%), 30 entre seis e oito anos $(48,4 \%)$ e 24 entre nove e 12 anos $(38,7 \%)$. A média de idade foi de $7,7 \pm 2,2$ anos, sendo a mediana equivalente 8 anos. Em ambos os grupos a distribuição da idade é normal, não havendo diferença significante pelo teste Qui- quadrado $(\mathrm{p}=0,58)$.

A mediana da duração da terapia fonoaudiológica foi de seis meses. Quanto ao número total de sessões, ao número de atendimentos e ao número de faltas verificou-se mediana de 17, 12 e 4,5 respectivamente. Não foi observada diferença significante entre os grupos em relação a essas variáveis. Estes dados, assim como os que se referem à distribuição dos indivíduos quanto ao número de atrasos, podem ser verificados na Tabela 1.

Para a transformação das variáveis contínuas em categóricas quanto à duração da terapia em meses, número total de sessões, de atendimentos e de faltas, estabeleceu-se como ponto de corte a mediana obtida para cada uma das variáveis: seis meses, total de 17 sessões, 12 atendimentos e 4,5 faltas respectivamente. Os dados são apresentados na Tabela 2, não tendo sido aferida diferença estatística entre os grupos.

A Figura 1 indica os achados relativos à distribuição dos indivíduos quanto à realização dos procedimentos indicados pelo terapeuta. Observou-se que menos da metade dos sujeitos realizava as orientações fonoaudiológicas $(41,2 \%)$, não havendo diferença significante entre os grupos quanto a esse parâmetro.

Os dados sobre a conclusão da terapia podem ser verificados na Figura 2. Constatou-se que o desligamento foi o desfecho mais frequente da terapia em ambos os grupos $(73,3 \%)$. Novamente, não foi identificada diferença significante entre os grupos.

Os motivos de desligamento são apresentados na Tabela 3, na qual se verifica, no total, predominância do excesso de faltas $(24,4 \%)$. Entretanto, analisando-se os grupos separadamente é possível perceber que a falta de compromisso $(17,9 \%)$, a espera por intervenção ortodôntica/otorrinolaringológica $(17,9 \%)$ e o excesso de faltas $(10,7 \%)$ predominam no Grupo MO; e o excesso de faltas $(30,6 \%)$, a falta de comprometimento $(11,6 \%)$, e o excesso de faltas associado a dificuldades financeiras $(9,7 \%)$, no Grupo RO. Verificou-se diferença estatisticamente significante entre os grupos em relação ao excesso de faltas $(\mathrm{p}<0,01)$, predominante no Grupo $\mathrm{RO}$ e à necessidade de se interromper o atendimento para se aguardar intervenção ortodôntica ou otorrinolaringológica, $(\mathrm{p}<0,01)$, predominante no Grupo MO.

A Tabela 4 apresenta a associação entre a distribuição categórica das faltas e conclusão da terapia, sendo que, no Grupo RO, foi verificada associação significante entre estes parâmetros $(\mathrm{p}<0,01)$.

Observa-se, na Tabela 5, a associação entre as variáveis 
Tabela 1. Distribuição dos indivíduos quanto à duração da terapia, presença e pontualidade às sessões

\begin{tabular}{|c|c|c|c|c|c|}
\hline & & \multicolumn{3}{|c|}{ Grupos } & \multirow{2}{*}{ Valor de $\mathrm{p}^{*}$} \\
\hline & & $\mathrm{MO}$ & $\mathrm{RO}$ & Total & \\
\hline \multirow[t]{5}{*}{ Meses de terapia } & Média & 8,12 & 6,73 & 7,65 & 0,79 \\
\hline & Desvio padrão & 7,07 & 4,48 & 2,39 & \\
\hline & Mediana & 6,00 & 6,25 & 6,00 & \\
\hline & Mínimo & 0,25 & 0,50 & 0,25 & \\
\hline & Máximo & 25,75 & 25,75 & 17,00 & \\
\hline \multirow[t]{5}{*}{ Total de sessões } & Média & 25,1 & 20,6 & 22,0 & 0,97 \\
\hline & Desvio padrão & 21,8 & 12,2 & 15,8 & \\
\hline & Mediana & 16,5 & 17,5 & 17,0 & \\
\hline & Mínimo & 3 & 1 & 1 & \\
\hline & Máximo & 75 & 47 & 75 & \\
\hline \multirow[t]{5}{*}{ Atendimentos } & Média & 20,8 & 14,9 & 16,8 & 0,37 \\
\hline & Desvio padrão & 18,0 & 11,0 & 13,7 & \\
\hline & Mediana & 13,0 & 12,0 & 12,0 & \\
\hline & Mínimo & 1 & 1 & 1 & \\
\hline & Máximo & 56 & 44 & 56 & \\
\hline \multirow[t]{5}{*}{ Faltas } & Média & 4,2 & 5,6 & 5,2 & 0,06 \\
\hline & Desvio padrão & 4,8 & 4,3 & 4,5 & \\
\hline & Mediana & 3,0 & 5,0 & 4,5 & \\
\hline & Mínimo & 0 & 0 & 0 & \\
\hline & Máximo & 19 & 18 & 19 & \\
\hline \multirow[t]{5}{*}{ Atrasos } & Média & 0,9 & 0,3 & 0,5 & 0,08 \\
\hline & Desvio padrão & 1,7 & 0,7 & 1,2 & \\
\hline & Mediana & 0,0 & 0,0 & 0,0 & \\
\hline & Mínimo & 0 & 0 & 0 & \\
\hline & Máximo & 7 & 3 & 7 & \\
\hline
\end{tabular}

Legenda: $\mathrm{MO}=$ grupo motricidade orofacial; $\mathrm{RO}=$ grupo respirador oral. * Teste Mann- Whitney

Tabela 2. Distribuição categórica dos indivíduos quanto duração da terapia em meses, ao número total de sessões, ao número de atendimentos e ao número de faltas

\begin{tabular}{|c|c|c|c|c|c|c|c|c|}
\hline & & \multicolumn{6}{|c|}{ Grupos } & \multirow{3}{*}{ Valor de $\mathrm{p}^{*}$} \\
\hline & & \multicolumn{2}{|l|}{$\mathrm{MO}$} & \multicolumn{2}{|c|}{ RO } & \multicolumn{2}{|l|}{ Total } & \\
\hline & & $\mathrm{N}$ & $\%$ & $\mathrm{~N}$ & $\%$ & $\mathrm{~N}$ & $\%$ & \\
\hline \multirow[t]{2}{*}{ Meses de terapia } & $\leq 6$ & 15 & 53,6 & 30 & 48,4 & 45 & 50,0 & 0,70 \\
\hline & $>6$ & 13 & 46,4 & 32 & 51,6 & 45 & 50,0 & \\
\hline \multirow[t]{2}{*}{ Total de sessões } & $\leq 17$ & 15 & 53,6 & 31 & 50,0 & 46 & 51,1 & 0,75 \\
\hline & $>17$ & 13 & 46,4 & 31 & 50,0 & 44 & 48,9 & \\
\hline \multirow[t]{2}{*}{ Atendimentos } & $\leq 12$ & 14 & 50,0 & 35 & 56,5 & 49 & 54,4 & 0,57 \\
\hline & $>12$ & 14 & 50,0 & 27 & 43,5 & 41 & 45,6 & \\
\hline \multirow[t]{2}{*}{ Faltas } & $\leq 4,5$ & 18 & 64,4 & 27 & 43,5 & 45 & 50,0 & 0,07 \\
\hline & $>4,5$ & 10 & 35,7 & 35 & 56,5 & 45 & 50,0 & \\
\hline
\end{tabular}

Legenda: $\mathrm{MO}$ = grupo motricidade orofacial; $\mathrm{RO}=$ grupo respirador oral. * Teste Qui-quadrado

adesão e conclusão da terapia, sendo que para esta análise foram excluídos os pacientes que compareceram a cinco ou menos sessões. Novamente no Grupo RO foi aferida associação estatística entre os aspectos estudados $(\mathrm{p}<0,01)$.

A associação entre adesão ao tratamento e gênero é demonstrada na Figura 3, na qual constatou-se p-valor $(\mathrm{p}=0,06)$ bastante próximo ao ponto de corte no Grupo RO. Nessa análise também foram excluídos os pacientes que compareceram a cinco ou menos sessões.

\section{DISCUSSÃO}

Apesar da literatura médica acerca da adesão salientar os aspectos relativos à prescrição de medicamentos, o termo refere-se igualmente a um amplo espectro de ações em saúde que incluem as terapias; práticas específicas para aliviar, monitorar e gerenciar as doenças e seus sintomas; assim como estratégias de promoção e prevenção em saúde ${ }^{(2)}$. Deste modo, verifica-se que a discussão quanto à adesão também no campo da Fonoaudiologia é bastante apropriada e necessária.

No presente trabalho a mediana da duração da terapia em meses foi bastante próxima comparando-se o Grupo MO e o Grupo RO, entretanto distinta de estudo que verificou tempo médio de dois meses e meio ${ }^{(13)}$. Deve-se ressaltar, porém, que o objetivo do estudo citado era alcançar o selamento labial, exclusivamente, diferença metodológica a qual impede a 


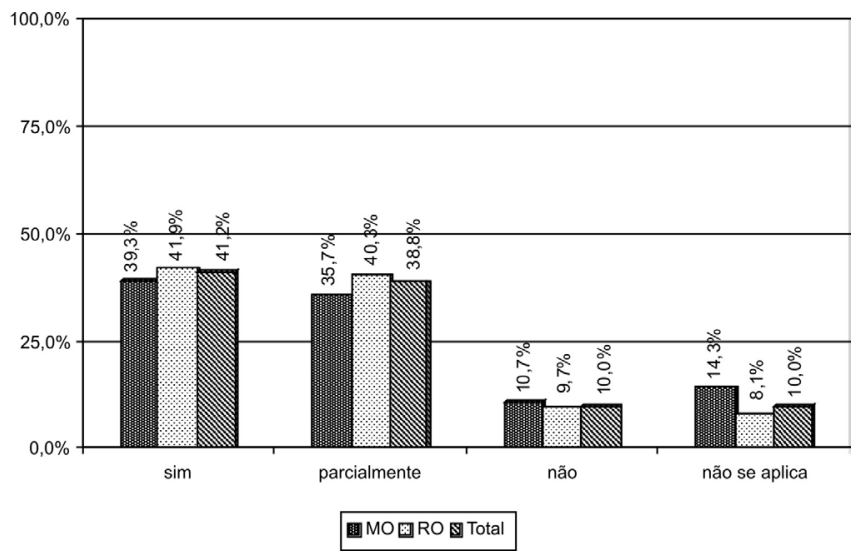

Legenda: $\mathrm{MO}$ = grupo motricidade orofacial; $\mathrm{RO}$ = grupo respirador oral

Figura 1. Distribuição dos indivíduos quanto à realização dos procedimentos indicados pelo terapeuta $(\mathrm{N}=90)$. Teste Qui-quadrado: $p=1,00$

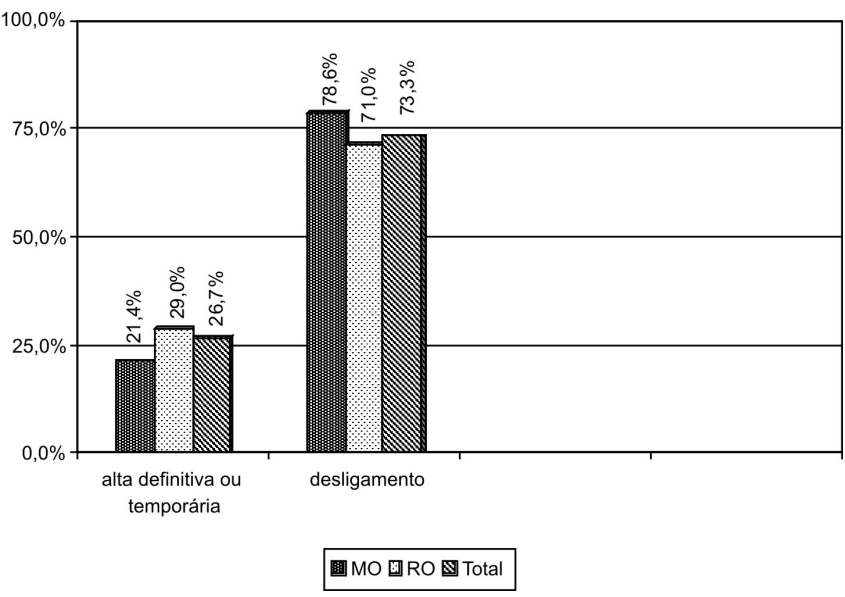

Legenda: $\mathrm{MO}$ = grupo motricidade orofacial; $\mathrm{RO}$ = grupo respirador oral

Figura 2. Distribuição dos indivíduos quanto à conclusão da terapia $(\mathrm{N}=90)$. Teste Qui-quadrado: $\mathrm{p}=0,45$

Tabela 3. Distribuição dos indivíduos quanto aos motivos de desligamento da terapia

\begin{tabular}{|c|c|c|c|c|c|c|c|}
\hline \multirow{3}{*}{ Motivos de desligamento da terapia } & \multicolumn{6}{|c|}{ Grupos } & \multirow{3}{*}{ Valor de $\mathrm{p}^{\star}$} \\
\hline & \multicolumn{2}{|l|}{$\mathrm{MO}$} & \multicolumn{2}{|l|}{ RO } & \multicolumn{2}{|c|}{ Total } & \\
\hline & $\mathrm{N}$ & $\%$ & $\mathrm{~N}$ & $\%$ & $\mathrm{~N}$ & $\%$ & \\
\hline Excesso de faltas & 3 & 10,7 & 19 & 30,6 & 22 & 24,4 & $<0,01$ \\
\hline Falta de compromisso & 5 & 17,9 & 7 & 11,3 & 12 & 13,3 & 0,39 \\
\hline Incompatibilidade de horários & 2 & 7,1 & 2 & 3,2 & 4 & 4,4 & - \\
\hline Sem acompanhante para trazer & 1 & 3,6 & 1 & 1,6 & 2 & 2,2 & - \\
\hline Sem justificativa & 0 & 0,0 & 1 & 1,6 & 1 & 1,1 & - \\
\hline Aguarda intervenção ortod. ou ORL & 5 & 17,9 & 0 & 0,0 & 5 & 5,5 & $<0,01$ \\
\hline Limite terapêutico & 1 & 3,6 & 2 & 3,2 & 3 & 3,3 & - \\
\hline Sem demanda & 2 & 7,1 & 0 & 0,0 & 2 & 2,2 & - \\
\hline Paciente imaturo & 1 & 3,6 & 0 & 0,0 & 1 & 1,1 & - \\
\hline Excesso de faltas e falta de compromisso & 1 & 3,6 & 5 & 8,1 & 6 & 6,6 & 0,43 \\
\hline Excesso de faltas e dificuldades financeiras & 0 & 0,0 & 6 & 9,7 & 6 & 6,6 & 0,09 \\
\hline Excesso de faltas, dif. fin. e s/ acompanh. & 0 & 0,0 & 1 & 1,6 & 1 & 1,1 & - \\
\hline Falta de compromisso e aguarda orto. & 1 & 3,6 & 0 & 0,0 & 1 & 1,1 & - \\
\hline Total & 22 & 78,6 & 44 & 71,0 & 66 & 73,3 & \\
\hline
\end{tabular}

Legenda: $\mathrm{MO}$ = grupo motricidade orofacial; $\mathrm{RO}$ = grupo respirador oral; Aguarda intervenção ortod. ou ORL = aguarda intervenção ortodôntica ou otorrinolaringológica; Dif. fin. e s/ acompanh. = dificuldades financeiras e sem acompanhante; Aguarda orto. = aguarda intervenção ortodôntica; - = não foi possível ou pertinente calcular o p-valor. * Teste Qui-quadrado

Tabela 4. Associação entre as variáveis distribuição categórica das faltas e conclusão da terapia

\begin{tabular}{|c|c|c|c|c|c|c|c|}
\hline & & \multicolumn{6}{|c|}{ Distribuição categórica das faltas } \\
\hline & & \multicolumn{3}{|c|}{ Grupo MO } & \multicolumn{3}{|c|}{ Grupo RO } \\
\hline & & $\leq 4,5$ & $>4,5$ & Total & $\leq 4,5$ & $>4,5$ & Total \\
\hline \multirow[t]{2}{*}{ Altas } & $\mathrm{N}$ & 5 & 1 & 6 & 13 & 5 & 18 \\
\hline & $\%$ & 17,8 & 3,6 & 21,4 & 21,0 & 8,0 & 29,0 \\
\hline \multirow[t]{2}{*}{ Desligamentos } & $\mathrm{N}$ & 13 & 9 & 22 & 14 & 30 & 44 \\
\hline & $\%$ & 46,5 & 32,1 & 78,6 & 22,6 & 48,4 & 71,0 \\
\hline \multirow[t]{2}{*}{ Total } & $\mathrm{N}$ & 18 & 10 & 28 & 27 & 35 & 62 \\
\hline & $\%$ & 64,3 & 35,7 & 100,0 & 43,6 & 56,4 & 100,0 \\
\hline Valor de $\mathrm{p}^{*}$ & & & 0,27 & & & $<0,01$ & \\
\hline
\end{tabular}

Legenda: $\mathrm{MO}=$ grupo motricidade orofacial $; \mathrm{RO}=$ grupo respirador oral.

* Teste Qui-quadrado comparação direta dos resultados com os do presente estudo, em que a terapia visava objetivos variados em relação ao aprimoramento/adequação do sistema estomatognático. É importante destacar que houve ampla variação quanto à duração da terapia observando-se, entretanto, que os valores mínimos e máximos são muito próximos em ambos os grupos.

Em relação à distribuição categórica dos indivíduos quanto à duração da terapia em meses, houve ligeira prevalência, em ambos os grupos, da duração menor ou igual a seis meses. Este tempo pode ser considerado adequado levando-se em conta o fato de que os terapeutas são graduandos, apresentando, portanto, pouca experiência clínica para serem hábeis a minimizar a duração do processo terapêutico. Além disso, ocorre rotatividade de terapeutas a cada semestre, fato que também atrasa a evolução do processo terapêutico, uma vez que algumas sessões são empregadas em processos reavaliativos. 
Tabela 5. Associação entre as variáveis adesão e conclusão da terapia

\begin{tabular}{|c|c|c|c|c|c|c|c|c|c|}
\hline \multirow{3}{*}{ Conclusão da terapia } & & \multicolumn{8}{|c|}{ Adesão } \\
\hline & & \multicolumn{4}{|c|}{ Grupo MO } & \multicolumn{4}{|c|}{ Grupo RO } \\
\hline & & Sim & Parcial & Não & Total & Sim & Parcial & Não & Total \\
\hline \multirow[t]{2}{*}{ Altas } & $\mathrm{N}$ & 4 & 1 & 1 & 6 & 16 & 2 & 0 & 18 \\
\hline & $\%$ & 16,6 & 4,2 & 4,2 & 25,0 & 28,1 & 3,6 & 0,0 & 31,7 \\
\hline \multirow[t]{2}{*}{ Desligamentos } & $\mathrm{N}$ & 7 & 9 & 2 & 18 & 10 & 23 & 6 & 39 \\
\hline & $\%$ & 29,2 & 37,5 & 8,3 & 75,0 & 17,5 & 40,3 & 10,5 & 68,3 \\
\hline \multirow[t]{2}{*}{ Total } & $\mathrm{N}$ & 11 & 10 & 3 & 24 & 26 & 25 & 6 & 57 \\
\hline & $\%$ & 45,8 & 41,7 & 12,5 & 100,0 & 45,6 & 43,8 & 10,6 & 100,0 \\
\hline Valor de $\mathrm{p}^{*}$ & & \multicolumn{4}{|c|}{0,36} & \multicolumn{4}{|c|}{$<0,01$} \\
\hline
\end{tabular}

Legenda: $\mathrm{MO}$ = grupo motricidade orofacial ; $\mathrm{RO}=$ grupo respirador oral. * Teste Qui-quadrado

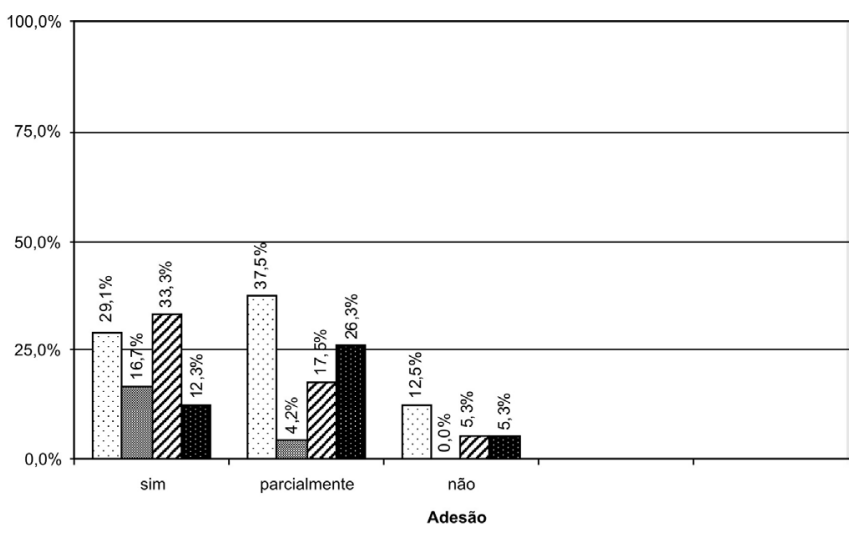

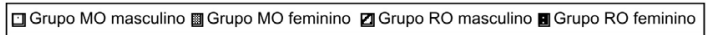

Legenda: $\mathrm{MO}$ = grupo motricidade orofacial; $\mathrm{RO}$ = grupo respirador oral

Figura 3. Associação entre as variáveis adesão e gênero ( $N=81)$. Teste Qui-quadrado: $p=0,21$ (Grupo MO); $p=0,06$ (Grupo RO)

Considerando-se a alta prevalência de desligamentos encontradas neste estudo, os dados apresentados quanto à duração da terapia em meses não indicam necessariamente o tempo para se alcançar a alta, mas sim a duração do período em que os pacientes permaneceram sob intervenção fonoaudiológica.

No que concerne ao número total de sessões, não houve diferença estatisticamente significante entre os grupos. Observou-se desvio padrão amplo, indicando dados heterogêneos, apesar de os valores da mediana serem muito semelhantes entre os grupos. O número máximo de sessões foi maior no Grupo MO, chegando a 75, contudo constatouse número elevado também no Grupo RO, equivalente a 47 sessões. Na literatura pesquisada não foram encontradas referências relativas ao número total de sessões oferecidas.

Quanto à distribuição categórica dos indivíduos relativa a este mesmo aspecto, constatou-se no Grupo MO discreta prevalência de 17 ou menos sessões oferecidas, enquanto no Grupo RO, a distribuição foi paritária entre os pacientes com mais e menos que 17 sessões. Estes dados se referem às sessões que realmente ocorreram e às que os pacientes faltaram, fato bastante frequente. Por conseguinte, os resultados não se referem à quantidade de sessões realizadas e devem ser analisados com critério.

Na literatura, são encontrados relatos de três a 39 sessões como o tempo necessário para conclusão da terapia ${ }^{(21)}$, bem como tempo médio de nove sessões para concluir a terapia com o objetivo único de automatizar o selamento labial ${ }^{(13)}$. Outro estudo verificou que o número médio de sessões em motricidade orofacial até a alta foi equivalente a 25,1 sessões para as crianças; 22,8 para adolescentes e 28,9 para adultos ${ }^{(12)}$. Considerando-se o valor da mediana para o número de atendimentos realizados, os achados observados no presente estudo se assemelham ao primeiro e segundo citados, divergindo dos constatados pelo terceiro. Em relação à distribuição categórica dos indivíduos quanto ao número de atendimentos realizados, prevaleceu no Grupo RO mediana menor ou igual a 12 sessões. No Grupo MO, ocorreu número igual de sujeitos com mais e menos de 12 atendimentos, não tendo sido verificada diferença estatística entre os grupos.

No que concerne ao número de faltas, não houve diferença significativa entre os grupos. No entanto, observase que o valor de $(p)$ foi próximo ao ponto de corte $(\mathrm{p}=0,06)$, indicando uma tendência a ocorrerem mais faltas no Grupo RO. Quanto à distribuição categórica dos indivíduos relativa às faltas, no Grupo RO houve predomínio da ocorrência de mais que 4,5 faltas, enquanto no Grupo MO, verificou-se o oposto. Novamente, observou-se valor de (p) próximo a 0,05 $(\mathrm{p}=0,07)$. Estes valores reiteram a hipótese de que o acesso diferenciado dos grupos ao atendimento fonoaudiológico implica em comportamentos de adesão, dentre eles a assiduidade, diferenciados. Uma vez que o Grupo MO realiza uma busca mais ativa pelo atendimento, evitaria mais as faltas em comparação ao Grupo RO. Não foram encontrados, na literatura compilada, dados acerca do número de faltas.

Em relação ao número de atrasos, verificou-se baixa ocorrência em ambos os grupos. Entretanto, essas informações pouco aparecem nos prontuários e, portanto, os dados aferidos no presente estudo contradizem a realidade observada no Ambulatório de Fonoaudiologia do HC/UFMG. É fundamental que os terapeutas se conscientizem da necessidade de descrever nos prontuários dados relativos aos atrasos, já que estes se configuram em importantes indicadores da adesão à fonoterapia. Não foram encontrados, na literatura compilada, estudos referentes a este aspecto. Além disso, não foi apresentada a distribuição categórica dos indivíduos quanto ao número de atrasos, devido à frequente ausência de informação quanto a esse aspecto nos prontuários.

No presente estudo verificou-se que menos da metade dos pacientes, em ambos os grupos, realizavam satisfatoriamente os procedimentos indicados pelo terapeuta, correspondendo 
a 39,3\% do Grupo MO e 41,9\% do Grupo RO. Foi observada prevalência discretamente menor de sujeitos que os realizavam apenas parcialmente, equivalente a 35,7\% do Grupo $\mathrm{MO}$ e 40,3\% do Grupo RO. Não foi observada diferença estatística entre os grupos. Estes achados não corroboram os de um estudo, realizado em clínica particular, em que, dentre os pacientes em alta fonoaudiológica na área de motricidade orofacial, houve maior prevalência de pacientes colaboradores, equivalente a $70 \%$ das crianças, $68 \%$ dos adolescentes e $100 \%$ dos adultos ${ }^{(12)}$. Em concordância com este achado, uma pesquisa de metanálise constatou que a adesão é significativamente maior em amostras de pacientes adultos, sendo que a média de não adesão foi de $25 \%{ }^{(22)}$. As diferenças metodológicas entre os estudos podem ser a causa dos achados divergentes, pois os estudos mencionados se referem a pacientes de clínica particular e que receberam alta. Em contrapartida, a presente pesquisa refere-se apenas a pacientes atendidos no sistema público de saúde e considerou também pacientes desligados da terapia. Ademais, a literatura compilada refere-se a crianças, adolescentes e adultos e os sujeitos do presente estudo são exclusivamente crianças de três a 12 anos de idade.

Em relação à conclusão da terapia fonoaudiológica, um estudo realizado com adultos apresentando queixas de fala verificou prevalência de alta definitiva ou controlada de 57\% e percentual de pacientes que desistiram da terapia equivalente à $32 \%{ }^{(23)}$. Outro estudo, com amostra de pacientes de uma clínica particular, constatou que 54\% dos pacientes na área de motricidade orofacial receberam alta ${ }^{(12)}$. Estes achados diferem dos constatados no presente estudo em que pequena parcela da amostra recebeu alta definitiva ou temporária, verificando-se prevalência de desligamentos extremamente elevada (73,3\%), novamente sem diferenças entre os grupos. É importante destacar que os estudos apresentam características metodológicas divergentes quando comparadas à presente pesquisa, a qual apresenta em sua amostra apenas crianças, com queixas variadas, atendidas pelo sistema público de saúde.

As barreiras citadas na literatura para a adesão à atividade física incluem fatores demográficos, psicológicos, socioculturais, ambientais, idade ${ }^{(24)}$ assim como o nível socioeconômico $^{(24-25)} \mathrm{e}$ a falta de tempo ${ }^{(26)}$. Adultos com queixas de fala relatam como motivos para a desistência da terapia a impossibilidade de comparecimento devido ao horário de trabalho (64\%) e satisfação com resultados parciais da terapia $(36 \%)^{(23)}$. Os achados da literatura, apesar de incluírem uma faixa etária diferente, corroboram em parte os verificados no presente estudo, em que os motivos de desligamento mais recorrentes foram a falta de compromisso, espera por intervenção ortodôntica/otorrinolaringológica e o excesso de faltas no Grupo MO; e o excesso de faltas, excesso de faltas associado à falta de comprometimento, e o excesso de faltas associado a dificuldades financeiras no Grupo RO, sendo este último motivo também referido por outros autores ${ }^{(24-25)}$.

Foi verificada diferença estatisticamente significante entre os grupos em relação à faltas excessivas como motivo de desligamento $(\mathrm{p}=0,01)$. Acredita-se que o excesso de faltas predominou no Grupo RO devido ao fato destes pacientes procurarem o ambulatório do Respirador Oral com a expectativa de serem avaliados e tratados por outros profissionais, tais como o otorrinolaringologista e o alergologista. Contudo, após avaliação e conduta da equipe interdisciplinar, eles e suas famílias são informados da necessidade do atendimento fonoaudiológico, fato que muitas vezes os surpreende. Ademais, o próprio serviço, devido à proximidade física e facilidade administrativa, marca o início da terapia em curto espaço de tempo. Em contrapartida, no Grupo MO os sujeitos são encaminhados por diferentes profissionais, dos diversos níveis de atenção em saúde, e para conseguir o atendimento fonoaudiológico os familiares precisam buscar as Unidades Básicas de Saúde e, após encaminhamento destas, ir ao ambulatório de Fonoaudiologia solicitar o atendimento, o qual costuma ter início moroso devido à longa fila de espera. Portanto, os pacientes do Grupo MO realizam uma busca mais ativa do atendimento quando comparados ao Grupo RO, sendo assim, valorizariam mais o tratamento oferecido, evitando as faltas.

É importante destacar também que os pacientes ou responsáveis de ambos os grupos são orientados, ao início da intervenção fonoaudiológica, sobre a importância da frequência e pontualidade às sessões e assinam um termo de compromisso demonstrando estar cientes em relação a estes aspectos. Neste termo é informado que ocorrendo três faltas o paciente será automaticamente excluído do atendimento e que casos especiais, de falta justificada com apresentação de atestado médico, serão analisados pelos supervisores que decidirão como proceder. Entretanto, mesmo diante do esclarecimento, observa-se que as faltas são muito frequentes em ambos os grupos.

Também foi constatada diferença estatisticamente significante entre os grupos em relação ao motivo de desligamento ser a espera por intervenção ortodôntica/ otorrinolaringológica $(\mathrm{p}<0,01)$. O referido motivo predominou no Grupo MO devido ao fato destes pacientes, quando precisam de encaminhamento para os profissionais mencionados, muitas vezes demoram a conseguir o tratamento e o realizam fora do complexo do Hospital das Clínicas. Já os pacientes do Grupo RO recebem o atendimento ortodôntico e otorrinolaringológico no próprio Ambulatório do Respirador Oral, geralmente prévio à intervenção fonoaudiológica.

Verificou-se, no Grupo RO, associação estatística entre a distribuição categórica das faltas e a conclusão da terapia $(p<0,01)$, sendo que o número de desligamentos foi significativamente maior entre os pacientes que apresentaram mais do que 4,5 faltas. Este achado indica que a assiduidade relaciona-se realmente com o sucesso da terapia, pois os pacientes menos frequentes foram os que mais concluíram a terapia com desligamento. Além disso, os dados reiteram as normas do ambulatório de Fonoaudiologia, que afirmam que caso os pacientes não sejam assíduos, perderão o direito ao tratamento. A associação entre os parâmetros estudados não foi aferida no Grupo MO. Não foram encontrados na literatura estudos que relacionassem as faltas à conclusão da terapia.

Quanto à associação entre a adesão e conclusão da terapia, observou-se que esta foi significante $(\mathrm{p}<0,01)$ novamente no Grupo RO, no qual o número de desligamentos foi maior entre os pacientes que realizavam as orientações dos terapeutas de forma apenas parcial. Ou seja, o comprometimento do 
paciente com os procedimentos terapêuticos determina o sucesso do tratamento e a continuidade deste em direção à alta ou a interrupção (desligamento). No Grupo MO, não ocorreu associação entre os aspectos mencionados. Contudo, em ambos os grupos, entre os pacientes que receberam alta ( $25 \%$ do Grupo MO e 31,7\% do Grupo RO), predominaram os que seguiam as orientações do terapeuta, correspondendo a 16,6\% do Grupo MO e 28,1\% do Grupo RO. Estes achados corroboram os de uma pesquisa, realizada em clínica particular, em que dentre os pacientes em alta fonoaudiológica na área de motricidade orofacial houve maior prevalência de pacientes colaboradores, equivalente à $70 \%$ das crianças ${ }^{(12)}$.

No que concerne a associação entre a adesão e gênero, esta apresentou p- valor bastante próximo ao ponto de corte $(\mathrm{p}=0,06)$ no Grupo $\mathrm{RO}$, no qual a adesão efetiva à terapia foi maior entre os indivíduos do sexo masculino, quando comparado ao feminino. Ressalta-se que este achado não era esperado uma vez que o número de pacientes do sexo masculino não foi significativamente maior do que os do sexo feminino. Na literatura pesquisada não foram mencionadas associações entre gênero e adesão.

Considerando-se os resultados do presente estudo ressaltase que era esperada maior diferença entre os grupos quanto aos dados de adesão e sucesso da terapia fonoaudiológica, uma vez que a forma de acesso dos pacientes é diferenciada. Acreditava-se que os sujeitos do Grupo MO, devido à busca ativa pela fonoterapia, seriam teoricamente mais conscientes de sua necessidade e, por conseguinte, mais assíduos e comprometidos com os procedimentos indicados, assim como teriam a alta fonoaudiológica como conclusão do processo terapêutico de forma mais prevalente, quando comparados ao Grupo RO.

Merece destaque também o fato de que muitos dados estavam ausentes nos prontuários dos pacientes, em ambos os grupos, sendo necessária, portanto, a conscientização dos terapeutas acerca deste aspecto. Esta característica foi um fator limitante na realização deste estudo, pois caso as informações dos pacientes fossem especificadas com mais frequência e melhor qualidade, os achados seriam mais fidedignos e contribuiriam mais efetivamente para a formulação de condutas diante do conhecimento amplo do perfil dos sujeitos atendidos.

Sugere-se que outros estudos abordando a caracterização em relação à adesão e sucesso da terapia fonoaudiológica em motricidade orofacial sejam conduzidos para que futuramente seja possível traçar o perfil destes pacientes, assim como formular condutas apropriadas às suas especificidades em níveis mais amplos, como, por exemplo, a esfera municipal. Acredita-se que, desta forma será aprimorada a qualidade e efetividade do atendimento oferecido a estes pacientes e, em consequência, melhores chances de acesso ao tratamento fonoaudiológico serão proporcionadas.

\section{CONCLUSÃO}

A análise dos prontuários dos pacientes atendidos no ambulatório de Fonoaudiologia do HC/UFMG indicou, considerando-se o cálculo da mediana, duração da terapia equivalente a 6 meses, número total de sessões igual a 17, número de atendimentos realizados 12 e número de faltas 4,5. A maioria dos pacientes realizava, de maneira integral ou parcial, os procedimentos indicados pelo terapeuta. Contudo, a conclusão da terapia foi majoritariamente o desligamento. A causa mais frequente de desligamento foi o excesso de faltas. Em consequência dessas características, o sucesso da terapia com adaptação/adequação das estruturas e funções do sistema estomatognático não foi alcançado na maioria dos casos.

Os pacientes que buscaram ativamente o ambulatório de Fonoaudiologia diferiram daqueles que foram encaminhados para fonoterapia pelo ambulatório do Respirador Oral quanto ao motivo de desligamento. Observou-se associação estatisticamente significante entre faltas e conclusão da terapia e entre adesão e conclusão da terapia, exclusivamente no Grupo RO.

\begin{abstract}
Purpose: To characterize orofacial myology treatment offered at the Ambulatory of Speech and Language Pathology of the Hospital das Clínicas of Universidade Federal de Minas Gerais concerning adhesion and therapy success, as well as to compare data from patients who actively searched for therapy and those referred by the Oral Breathing Ambulatory of the same institution. Methods: Ninety records of all patients that were discharged or resigned from treatment either at the Ambulatory of Speech and Language Pathology (MO Group) or at the Oral Breathing Ambulatory (RO Group) were investigated. The results were statistically analyzed using Mann-Whitney and Chi-square tests (5\%). Results: Median calculation showed that the duration of therapy was of 6,0 months, the total number of sessions was 17, the number of sessions carried out was 12 and the number of absences was equivalent to 4,5 . Only $41.2 \%$ of the patients carried out the procedures recommended by the therapist. The conclusion of the therapeutic process was mainly resignation (73.3\%), and excessive absences was most frequent reason for that $(24.4 \%)$. The groups differed from each other regarding resignation reason: absences predominated as the reason for the RO Group $(\mathrm{p}<0,01)$, while the wait for other professionals was the most frequent reason for the MO Group ( $\mathrm{p}<0,01)$. Significant associations regarding absences and resignation $(\mathrm{p}<0,01)$ and adhesion and resignation $(\mathrm{p}<0,01)$ were found in the RO Group. Conclusions: The researched characteristics were predominantly similar between the groups. Despite many patients followed recommendations totally or partially, adhesion was compromised by excessive absences. Thus, therapy success was not achieved in the majority of the cases studied.
\end{abstract}

Keywords: Speech therapy; Myofunctional therapy; Stomatognathic system abnormalities; Mouth breathing; Treatment outcome; Health services research; Patient discharge 


\section{REFERÊNCIAS}

1. Martins LM, Pimenta CA. Adesão do doente com dor crônica ao tratamento. Prat Hosp. 2000;2(11):7-11.

2. Sawyer SM, Aroni RA. Sticky issue of adherence. J Paediatr Child Health. 2003;39(1):2-5.

3. Irvine J, Baker B, Smith J, Jandciu S, Paquette M, Cairns J, et al. Poor adherence to placebo or amiodarone therapy predicts mortality: results from the CAMIAT study. Psychosom Med. 1999;61(4):566-75.

4. McDonald HP, Garg AX, Haynes RB. Interventions to enhance patient adherence to medication prescriptions: scientific review. JAMA. 2002;288(22):2868-79.

5. De Smet BD, Erickson SR, Kirking DM. Self-reported adherence in patients with asthma. Ann Pharmacother. 2006;40(3):414-20.

6. DiMatteo RM, Lepper HS, Croghan TW. Depression is a risk factor for noncompliance with medical treatment - Meta-analysis of the effects of anxiety and depression on patient adherence. Arch Intern Med. 2000;160(14):2101-7.

7. DiMatteo RM. Social support and patient adherence to medical treatment: a meta-analysis. Health Psychol. 2004;23(2):207-18.

8. Tesser CD, Luz MT. Racionalidades médicas e integralidade. Ciênc Saúde Coletiva. 2008;13(1):195-20.

9. Goulart BN, Chiari BM. Humanização das práticas do profissional de saúde - contribuições para reflexão. Ciênc Saúde coletiva [Internet] 2006 [citado 2008 Mar 18];11(3):[31 telas]. Disponível em URL: http://www.abrasco. org.br/cienciaesaudecoletiva/artigos/artigo_int. php?id_artigo $=960$

10. Haynes RB, McDonald HP, Garg AX. Helping patients follow prescribed treatment - clinical applications. JAMA. 2002;288(22):2880-3.

11. Skeat J, Perry A. Exploring the implementation and use of outcome measurement in practice: a qualitative study. Int J Lang Comm Dis. 2008;43(2):110-25.

12. Bacha SMC. Quantidade média de sessões para se dar alta em casos de motricidade orofacial [resumo]. Rev Soc Bras Fonoaudiol. 2005; Supl Especial.

13. Oliveira RO, Marchesan IQ, Araujo RLT, Costa MLVCM. Técnicas para diminuir o tempo médio de sessões para o fechamento labial [resumo]. Rev Soc Bras Fonoaudiol. 2006; Supl Especial.

14. Bianchini EM. Avaliação fonoaudiológica da motricidade oral distúrbios miofuncionais orofaciais ou situações adaptativas. Rev Dent
Press Ortodon Ortoped Facial. 2001;6(3):73-82.

15. Korbmacher H, Kahl-Nieke B. Optimizing interdisciplinary cooperation for patients with orofacial dysfunctions. Presentation of an interdisciplinary diagnostic referral sheet. J Orofac Orthop. 2001;62(3):246-50.

16. Rodrigues HO, Faria SR, Paula FS, Motta AR. Ocorrência de respiração oral e alterações miofuncionais orofaciais em sujeitos em tratamento ortodôntico. Rev CEFAC. 2005;7(3):356-62.

17. Barros JR, Becker HM, Pinto JA. Avaliação de atopia em crianças respiradoras bucais atendidas em centro de referência. J Pediatr (Rio J). 2006;82(6):318-25.

18. De Menezes VA, Leal RB, Pessoa RS, Pontes RM. Prevalência e fatores associados à respiração oral em escolares participantes do projeto Santo Amaro-Recife, 2005. Rev Bras Otorrinolaringol. 2006;72(3):394-9.

19. Marchesan IQ. Protocolo de avaliação miofuncional orofacial. In: Krakauer LH, Di Francesco RC, Marchesan IQ. Respiração oral: abordagem interdisciplinar. São Jose dos Campos: Pulso; 2003. p.55-79.

20. César AM, Maksud SS. Caracterização da demanda de fonoaudiologia no serviço público municipal de Ribeirão das Neves - MG. Rev CEFAC. 2007;9(1):133-8.

21. Oliveira RO, Marchesan IQ, Araujo RL, Costa ML. O quê ocorre após a alta fonoaudiológica [resumo]. Rev Soc Bras Fonoaudiol. 2006; Supl Especial.

22. DiMatteo RM. Variations in patients' adherence to medical recommendations: a quantitative review of 50 years of research. Med Care. 2004;42(3):200-9.

23. Oliveira RO, Marchesan IQ, Araujo RL, Costa ML. Adesão dos pacientes adultos a fonoterapia [resumo]. Rev Soc Bras Fonoaudiol. 2006; Supl Especial.

24. Chinn DJ, White M, Harland J, Drinkwater C, Raybould S. Barriers to physical activity and socioeconomic position: implications for health promotion. J Epidemiol Community Health. 1999;53(3):191-2.

25. Tai SS, Gould M, Smith P, Iliffe S. Promoting physical activity in general practice: should prescribed exercise be free? J R Soc Med. 1999;92(2):65-7.

26. Ribeiro MA, Martins Mde M, Carvalho CR. The role of physician counseling in improving adherence to physical activity among the general population. São Paulo Med J. 2007:125(2);115-21.

Anexo 1. Roteiro de coleta dos dados de prontuário

1. Adesão ao tratamento:

- Data de início da terapia:

- Data de término da terapia:

- Número total de sessões:

- Número de atendimentos:

- Número de faltas:

- Número de atrasos:

- Realização dos procedimentos indicados pelo terapeuta responsável:

Sim ( ) Parcialmente ( ) Não ( )

2. Sucesso da terapia fonoaudiológica:

- Alta fonoaudiológica com adequação/adaptação das estruturas e funções miofuncionais orofaciais ou alta temporária:

Sim ( ) Não ( )

- Desligamento da terapia: Sim ( ) Não ( )

Razões: 